\title{
Two-Stage Hepatectomy Procedure to Treat Initially Unresectable Multiple Bilobar Colorectal Liver Metastases: Technical Aspects
}

\author{
Masato Narita Elie Oussoultzoglou Philippe Bachellier Edoardo Rosso \\ Patrick Pessaux Daniel Jaeck \\ Centre de Chirurgie Viscérale et de Transplantation, Hôpital de Hautepierre, Hôpitaux Universitaires de Strasbourg, \\ Strasbourg, France
}

\section{Key Words}

Portal vein embolization - Portal vein ligation .

Neoadjuvant chemotherapy • Postoperative morbidity •

Liver resection $\cdot$ Major hepatectomy

\begin{abstract}
A two-stage hepatectomy procedure is a therapeutic strategy for patients presenting with initially unresectable multiple and bilobar colorectal liver metastases in order to achieve a curative R0 resection. The main goal of this approach is to minimize the risk of postoperative liver failure resulting from a too small remnant liver after completing a curative resection. This procedure combines two sequential liver resections that involve perioperative chemotherapy and portal vein embolization. This article describes our standardized strategy of two-stage hepatectomy combined with portal vein embolization used over the last 15 years and discusses the alternative procedures as well as their respective advantages and drawbacks.

Copyright $\odot 2011$ S. Karger AG, Basel
\end{abstract}

\section{Introduction}

Hepatic resection for colorectal metastases, limited to the liver, has become the standard of care, and currently remains the only potentially curative therapy. Several reports have demonstrated long-term survival and no other treatment options have shown the same survival benefit [1]. However, only around $25 \%$ of these patients are initially resectable at the time of referral to a unit specialized in hepatobiliary surgery [2]. Among these patients, about $50 \%$ have multiple and bilobar metastases, ill-located nodules requiring multiple and technically demanding liver resections but still resectable in a single stage [3]. A two-stage hepatectomy procedure consists in combining two sequential liver resections when it is impossible to resect all liver metastases in a single procedure while preserving at least $30 \%$ of the functional liver volume to avoid postoperative lethal liver failure. In reality, twostage hepatectomy is more a multidisciplinary approach than a combination of two sequential liver resections, as it is often associated with perioperative systemic chemotherapy as well as with preoperative portal vein emboliza-

\section{KARGER}

Fax +4161306 1234

E-Mail karger@karger.ch

www.karger.com
(C) 2011 S. Karger AG, Basel

$0253-4886 / 11 / 0282-0121 \$ 38.00 / 0$

Accessible online at:

www.karger.com/dsu
Daniel Jaeck, MD, PhD, FRCS

Centre de Chirurgie Viscérale et de Transplantation, Hôpital de Hautepierre

Hôpitaux Universitaires de Strasbourg, Université de Strasbourg

Avenue Molière, FR-67098 Strasbourg Cedex (France)

Tel. +33 3881272 56, E-Mail Daniel.Jaeck@chru-strasbourg.fr 

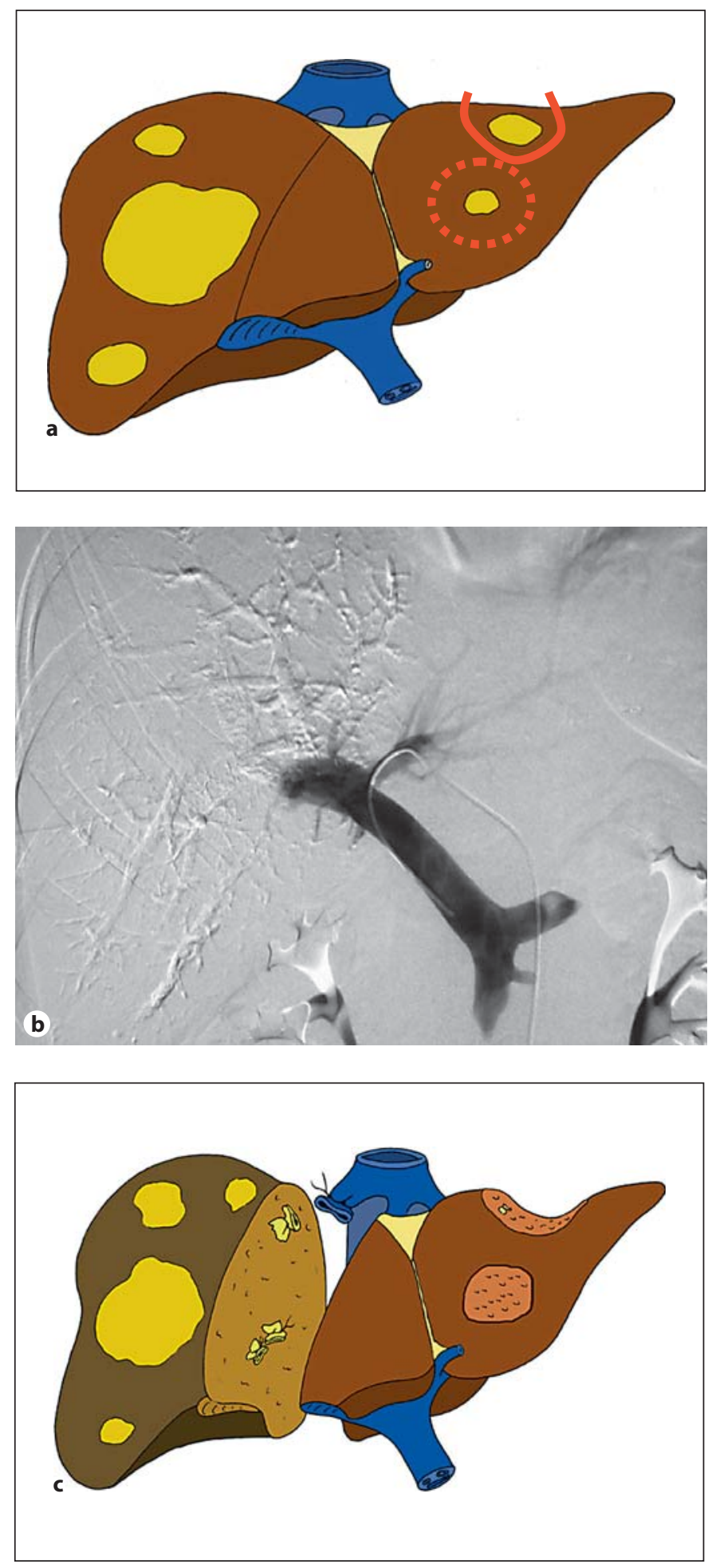

Fig. 1. a At the time of the 1st-stage hepatectomy, the left hemiliver is completely cleared from liver metastases by non-anatomical resections (encircled by a solid line) and by radiofrequency destruction (encircled by a dotted line). b PVE is performed with a percutaneous approach. c The 2nd-stage hepatectomy is performed with a curative intent. tion (PVE) [4]. Considering the different possibilities of combining these multidisciplinary therapeutic modalities, two main concepts to achieve two-stage hepatectomy have been described and used during the last decade in order to extend the frontiers of surgical therapy and to increase the number of patients presenting with initially unresectable colorectal liver metastases (CLM) who can benefit from hepatic resection. The aim of the present report is to define our standardized strategy of two-stage hepatectomy combined with PVE and to discuss the alternative procedures as well as their advantages and drawbacks.

\section{Patients and Methods}

\section{Patient Selection Criteria}

Candidates for two-stage hepatectomy are patients presenting with multiple and bilobar CLM which are initially considered as nonresectable, in whom a clearance of the left hemiliver is feasible during the 1st-stage hepatectomy, in whom the left hepatic pedicle and the left hepatic vein are not invaded by the tumor and not injured during the 1st-stage hepatectomy and in whom the future remnant liver volume is less than $30 \%$ of the total functional liver volume. Patients with jaundice (total bilirubin serum level $>50$ $\mu \mathrm{mol} / \mathrm{l})$, with altered liver function estimated by ICG-R15 (>20\%) and those presenting with unresectable extrahepatic abdominal disease are excluded.

\section{First-Stage Hepatectomy}

During the 1st-stage hepatectomy, the future remnant hemiliver, mostly the left, is cleared from all CLM (fig. 1a). Clearance is obtained by non-anatomical liver resections and/or radiofrequency destruction. These procedures are performed with or without intermittent hepatic pedicle clamping.

\section{Portal Vein Embolization}

Two to 4 weeks after the clearance of the future remnant hemiliver, a percutaneous transhepatic right PVE is performed (fig. 1b). Liver hypertrophy is estimated 4-8 weeks later by a threedimensional CT scan. When insufficient hypertrophy is obtained, a repeat volumetric evaluation is performed $3-4$ weeks later.

\section{Second-Stage Hepatectomy}

The 2nd-stage hepatectomy will be planned $4-8$ weeks after PVE following an appropriate hypertrophy of the nonembolized hemiliver. This second liver resection is mainly a right hepatectomy, sometimes extended to segment IV and aims to achieve a curative resection (fig. 1c).

\section{Perioperative Chemotherapy}

The decision to treat with chemotherapy is made by a multidisciplinary conference which includes oncologists, gastroenterologists, pathologists, and surgeons. Regarding preoperative chemotherapy, the most efficient chemotherapy regimen should be administered in order to offer to the patients the only chance of 
Table 1. Reported outcomes following two-stage hepatectomy in patients with CLM

\begin{tabular}{|c|c|c|c|c|c|c|c|c|c|}
\hline \multirow[t]{2}{*}{ First author } & \multirow[t]{2}{*}{ Year } & \multirow[t]{2}{*}{ Planned } & \multirow[t]{2}{*}{ Achieved } & \multicolumn{2}{|c|}{ Morbidity, \% } & \multicolumn{2}{|c|}{ Mortality, \% } & \multicolumn{2}{|c|}{ Survival, \% } \\
\hline & & & & 1 st & 2nd & 1 st & 2nd & 3 years & 5 years \\
\hline Adam & 2000 & 16 & 13 & 31 & 45 & 0 & 0 & 35 & NA \\
\hline Jaeck & 2004 & 33 & 25 & 15 & 56 & 0 & 0 & 54 & NA \\
\hline Togo & 2005 & 11 & 11 & 0 & 18 & 0 & 0 & 45 & NA \\
\hline Tanaka & 2007 & 24 & 22 & NA & 23 & 0 & 0 & 33 & NA \\
\hline Chun & 2007 & 30 & 21 & 24 & 43 & 0 & 0 & 86 & NA \\
\hline Pamecha & 2008 & 14 & 11 & 0 & 22 & 0 & 0 & NA & 50 \\
\hline Wicherts & 2008 & 59 & 41 & 20 & 59 & 0 & 7 & 60 & 42 \\
\hline Homayounfar & 2009 & 24 & 19 & 13 & 58 & 0 & 5 & NA & NA \\
\hline Tsai & 2010 & 45 & 35 & 24 & 26 & 4 & 5 & 58 & NA \\
\hline Karoui & 2010 & 33 & 25 & 21 & 32 & 0 & 4 & 80 & 48 \\
\hline
\end{tabular}

Planned = Number of patients planned to undergo two-stage hepatectomy; achieved = number of patients in whom two-stage hepatectomy was achieved; 1 st = 1st-stage hepatectomy; 2 nd = 2nd-stage hepatectomy; NA = not available.

cure. Computed tomography and magnetic resonance imaging are used to exclude disease progression before planning both 1stand 2nd-stage hepatectomy. A short period of chemotherapy (2-3 cycles) is currently administered between the 1st- and the 2ndstage hepatectomy; it usually starts 2-3 weeks after PVE.

\section{Results}

Since 1996, our 15-year-experience included a series of nearly 90 patients of whom around two thirds underwent two-stage hepatectomy combined with PVE. Postoperative mortality was nil. Postoperative morbidity after 1stand 2nd-stage hepatectomy occurred in around 10 and $40 \%$ of the patients, respectively.

\section{Discussion}

A two-stage hepatectomy procedure is a potentially curative multidisciplinary strategy, which may be applied only in highly selected patients presenting with initially unresectable CLM. Generally, almost all the patients scheduled for two-stage hepatectomy have been previously heavily treated with chemotherapy, while only a minority (less than 10\%) of the initially unresectable patients became eligible for a curative two-stage hepatectomy after administration of neoadjuvant chemotherapy, which also provides some drawbacks such as missing metastases and chemotherapy-induced hepatotoxicity [5-7]. Furthermore, the feasibility rate of the ini- tially planned two-stage hepatectomy was around two thirds of the patients, and the failure was mainly related to disease progression and/or to insufficient liver hypertrophy [5, 8-15]. However, completion of this procedure allows to provide, for patients presenting with multiple and bilobar CLM, a similar survival benefit to that reported in patients with initially resectable liver metastases (table 1) [3].

The small volume of the future remnant liver and the injury of the non-tumoral hepatic parenchyma resulting from preoperative chemotherapy, constitute the major obstacles to liver resection. The benefit of preoperative PVE has been demonstrated by a retrospective study [16], while the role of PVE in reducing the risk of liver failure after surgery is not yet clearly elucidated. Indeed, one prospective study showed that preoperative PVE before a right hepatectomy is not required in cases of normal non-tumoral hepatic parenchyma [17]. However, preoperative PVE before hepatectomy seems justified particularly when the remaining functional liver volume is less than 30 or $40 \%$ in patients receiving prolonged courses of preoperative chemotherapy [18]. Therefore, one-stage hepatectomy combined with PVE has been developed to reduce the risk of postoperative liver failure in patients presenting with liver metastases and requiring a right or extended right hepatectomy in the presence of a small left hemiliver even in patients with bilobar disease [19]. For these candidates, the left hemiliver and more particularly segments 2 and 3 must be completely free of metastases as PVE carries negative side effects particularly in patients with multiple and bilobar CLM. Indeed, 
PVE stimulates the proliferation of hepatocytes as well as the growth of occult metastases located in the nonembolized hemiliver which were undetectable by preoperative imaging [20]. As a consequence, progression of CLM in the remnant nonembolized hemiliver could preclude the achievement of a curative liver resection as previously demonstrated $[4,12]$. For this reason, CLM resection in the nonembolized hemiliver should precede the PVE. The rationale behind two-stage hepatectomy combined with PVE is to minimize the risk of CLM growth in the future remnant liver and also the risk of postoperative liver failure. After right PVE, a progressive atrophy of the right hemiliver and a compensatory hypertrophy of the left hemiliver usually occur within 3-6 weeks allowing to safely perform a major hepatectomy. The 2nd-stage hepatectomy (right or extended right) is performed 4-8 weeks after PVE. This strategy offers the patients a longterm survival similar to that obtained after liver resection in patients with initially resectable liver metastases and without the use of preoperative PVE (table 1) [5, 8-15].

Alternative strategies have also been developed. First, the 1st stage can be performed at the time of a laparoscopic removal of the primary colorectal tumor either by laparoscopic resection of the metastases within the left hemiliver or by the use of percutaneous radiofrequency ablation. However, percutaneous radiofrequency destruction may overlook the presence of occult CLM and of extrahepatic disease. Secondly, in order to induce hypertrophy of the contralateral hemiliver, a portal vein ligation (PVL) can be performed during the 1st-stage hepatectomy as an alternative to PVE, particularly in patients presenting with synchronous CLM. However, PVL seems to be less efficient than PVE as previously reported [21]. In fact, this should be further confirmed by a randomized study. Thirdly, the use of PVE is not always mandatory. Indeed, a longer waiting period between both hepatectomies may allow an adequate hypertrophy of the entire liver parenchyma to be reached. During this waiting period, patients often receive chemotherapy. In this strategy, during the first hepatectomy the highest number of liver metastases are resected, regardless of their location, and liver metastases left in place during the first hepatectomy will be resected during a second hepatectomy after a period of liver regeneration without the use of PVE. This scenario was first described by Adam et al. [5]. However, this approach will result in making it more difficult to perform the 2nd-stage hepatectomy because of adhesions of the large cut liver surface to the surrounding tissues including colon and diaphragm. Furthermore, this approach would also lead to the risk of tumor proliferation induced by increased level of growth factors within the remaining hemiliver during liver regeneration following major hepatectomy. Considering all these arguments, it seems logical to perform the clearance of metastases within the future remnant liver during the 1st-stage hepatectomy. Finally, an increased use of parenchyma-sparing surgery for bilobar CLM [22] as well as an increased use of mini-invasive procedures such as radiofrequency ablation resulted in a significant decrease in mortality after these combined procedures. In their experience, Gold et al. [22] reported a median number of liver metastases of 2 , and only $30 \%$ of the study population had 4 or more liver metastases. In such circumstances, it seems obvious and justified to avoid two-stage hepatectomy. Recently, Torzilli et al. [23] reported one-stage instead of two-stage hepatectomy for patients presenting with multiple and bilobar CLM. This one-stage approach was based on intraoperative ultrasound-guided resections. The authors mentioned that one-stage hepatectomy constitutes a parenchymasparing liver resection resulting in a safer surgery. However, this technique may have three main drawbacks; (1) the absence of resection margin reported in nearly half of the patients, (2) a prolonged time of hepatic pedicle clamping, and (3) a risk of overlooking small metastases undetectable by intraoperative ultrasound.

\section{Tumor Margin}

Recently, it has been reported that tumor margin was not associated with early tumor recurrence [24], and even an $\mathrm{R} 1$ resection was considered to be acceptable in the current era of effective chemotherapy [25]. However, there have also been some reports describing that tumor margin was an independent prognostic factor for overall and recurrence-free survival, and micrometastases detected within $1 \mathrm{~cm}$ separated from the gross tumor occurred in $58 \%$ of patients $[26,27]$. Therefore, this strategy still seems controversial.

\section{Prolonged Hepatic Pedicle Clamping}

The authors used prolonged intermittent clamping with Pringle maneuver (116 min of median clamping time, range 65-348) [23]. Several experimental studies demonstrated that ischemia-reperfusion injury strongly stimulated outgrowth of intrahepatic colorectal liver micrometastases [28, 29]. Furthermore, there has not yet been a long-term outcome reported for these patients undergoing such one-stage hepatectomy. 


\section{Risk for Overlooking Small Metastases}

The ability of imaging to detect small metastases seems questionable since Elias et al. [30] showed, in patients presenting with liver metastases from endocrine tumor, that the number of liver metastases was significantly higher on the pathologic examination of the specimen than that detectable by different preoperative and intraoperative imaging. Indeed, the accuracy for each examination in detecting liver metastases is less than $50 \%$. Therefore, it seems too early to conclude which strategy is the most suitable for patients with multiple bilobar CLM.

\section{Conclusion}

A significant evolution occurred during the last decade in the application of novel therapies leading to a more aggressive approach in the management of CLM. The use of innovative multidisciplinary approaches which integrate advanced surgical techniques as well as novel therapeutic options such as neoadjuvant chemotherapy, portal vein occlusion, local ablative therapy, and staged resection allowed to extend the indications of a curative intent hepatic resection in patients with initially unresectable liver metastases. Two main factors affect the success of two-stage hepatectomy: careful patient selection and adequate use of new optimal chemotherapy regimens. Although a two-stage hepatectomy procedure is not so frequently used, the demand for it will increase in the future because two-stage hepatectomy may be the only therapeutic strategy able to provide long-term survival and a possible cure for patients presenting with initially unresectable multiple and bilobar CLM.

\section{Disclosure Statement}

The authors have no conflicts of interest.

\section{References}

1 Simmonds PC, Primrose JN, Colquitt JL, et al: Surgical resection of hepatic metastases from colorectal cancer: a systematic review of published studies. Br J Cancer 2006;94: 982-999.

2 Minagawa M, Makuuchi M, Torzilli G, et al: Extension of the frontiers of surgical indications in the treatment of liver metastases from colorectal cancer: long-term results. Ann Surg 2000;231:487-499.

3 Fong Y, Fortner J, Sun RL, et al: Clinical score for predicting recurrence after hepatic resection for metastatic colorectal cancer: analysis of 1,001 consecutive cases. Ann Surg 1999;230:309-318, discussion 318-321.

4 Jaeck D, Oussoultzoglou E, Rosso E, et al: A two-stage hepatectomy procedure combined with portal vein embolization to achieve curative resection for initially unresectable multiple and bilobar colorectal liver metastases. Ann Surg 2004;240:1037-1049, discussion 1049-1051.

5 Adam R, Laurent A, Azoulay D, et al: Twostage hepatectomy: a planned strategy to treat irresectable liver tumors. Ann Surg 2000;232:777-785.

6 Benoist S, Brouquet A, Penna C, et al: Complete response of colorectal liver metastases after chemotherapy: does it mean cure? J Clin Oncol 2006;24:3939-3945.

7 Zorzi D, Laurent A, Pawlik TM, et al: Chemotherapy-associated hepatotoxicity and surgery for colorectal liver metastases. Br J Surg 2007;94:274-286.
-8 Togo S, Nagano Y, Masui H, et al: Two-stage hepatectomy for multiple bilobular liver metastases from colorectal cancer. Hepatogastroenterology 2005;52:913-919.

9 Chun YS, Vauthey JN, Ribero D, et al: Systemic chemotherapy and two-stage hepatectomy for extensive bilateral colorectal liver metastases: perioperative safety and survival. J Gastrointest Surg 2007;11:1498-1504, discussion 1504-1505.

10 Tanaka K, Shimada H, Matsuo K, et al: Remnant liver regeneration after two-stage hepatectomy for multiple bilobar colorectal metastases. Eur J Surg Oncol 2007;33:329-335.

11 Pamecha V, Nedjat-Shokouhi B, Gurusamy $\mathrm{K}$, et al: Prospective evaluation of two-stage hepatectomy combined with selective portal vein embolisation and systemic chemotherapy for patients with unresectable bilobar colorectal liver metastases. Dig Surg 2008; 25:387-393.

12 Wicherts DA, Miller R, de Haas RJ, et al: Long-term results of two-stage hepatectomy for irresectable colorectal cancer liver metastases. Ann Surg 2008;248:994-1005.

13 Homayounfar K, Liersch T, Schuetze G, et al: Two-stage hepatectomy (R0) with portal vein ligation - towards curing patients with extended bilobular colorectal liver metastases. Int J Colorectal Dis 2009;24:409-418.
14 Tsai S, Marques HP, de Jong MC, et al: Twostage strategy for patients with extensive bilateral colorectal liver metastases. HPB (Oxford) 2010;12:262-269.

15 Karoui M, Vigano L, Goyer P, et al: Combined first-stage hepatectomy and colorectal resection in a two-stage hepatectomy strategy for bilobar synchronous liver metastases. Br J Surg DOI: 10.1002/bjs.7128.

16 Covey AM, Brown KT, Jarnagin WR, et al: Combined portal vein embolization and neoadjuvant chemotherapy as a treatment strategy for resectable hepatic colorectal metastases. Ann Surg 2008;247:451-455.

17 Farges O, Belghiti J, Kianmanesh R, et al: Portal vein embolization before right hepatectomy: prospective clinical trial. Ann Surg 2003;237:208-217.

18 Adam R, Miller R, Pitombo M, et al: Twostage hepatectomy approach for initially unresectable colorectal hepatic metastases. Surg Oncol Clin North Am 2007; 16:525536, viii.

19 Kawasaki S, Makuuchi M, Kakazu T, et al: Resection for multiple metastatic liver tumors after portal embolization. Surgery 1994;115:674-677.

20 Elias D, De Baere T, Roche A, et al: During liver regeneration following right portal embolization the growth rate of liver metastases is more rapid than that of the liver parenchyma. Br J Surg 1999;86:784-788. 
21 Broering DC, Hillert C, Krupski G, et al: Portal vein embolization vs. portal vein ligation for induction of hypertrophy of the future liver remnant. J Gastrointest Surg 2002; 6:905-913; discussion 913.

22 Gold JS, Are C, Kornprat P, et al: Increased use of parenchymal-sparing surgery for bilateral liver metastases from colorectal cancer is associated with improved mortality without change in oncologic outcome: trends in treatment over time in 440 patients. Ann Surg 2008;247:109-117.

23 Torzilli G, Procopio F, Botea F, et al: Onestage ultrasonographically guided hepatectomy for multiple bilobar colorectal metastases: a feasible and effective alternative to the 2-stage approach. Surgery 2009;146:60-71.
24 Pawlik TM, Scoggins CR, Zorzi D, et al: Effect of surgical margin status on survival and site of recurrence after hepatic resection for colorectal metastases. Ann Surg 2005;241: 715-722, discussion 722-724.

25 de Haas RJ, Wicherts DA, Flores E, et al: R1 resection by necessity for colorectal liver metastases: is it still a contraindication to surgery? Ann Surg 2008;248:626-637.

26 Wakai T, Shirai Y, Sakata J, et al: Appraisal of $1 \mathrm{~cm}$ hepatectomy margins for intrahepatic micrometastases in patients with colorectal carcinoma liver metastasis. Ann Surg Oncol 2008; 15:2472-2481.

27 Nuzzo G, Giuliante F, Ardito F, et al: Influence of surgical margin on type of recurrence after liver resection for colorectal metastases: a single-center experience. Surgery 2008; $143: 384-393$.
28 Nicoud IB, Jones CM, Pierce JM, et al: Warm hepatic ischemia-reperfusion promotes growth of colorectal carcinoma micrometastases in mouse liver via matrix metalloproteinase-9 induction. Cancer Res 2007;67: $2720-2728$

29 van der Bilt JD, Kranenburg O, Nijkamp $\mathrm{MW}$, et al: Ischemia/reperfusion accelerates the outgrowth of hepatic micrometastases in a highly standardized murine model. Hepatology 2005;42:165-175.

30 Elias D, Lefevre JH, Duvillard P, et al: Hepatic metastases from neuroendocrine tumors with a 'thin slice' pathological examination: they are many more than you think. Ann Surg 2010;251:307-310. 